\title{
Uso de las tecnologías de la información y comunicación (TIC) en las residencias médicas en México
}

\author{
Gerhard Heinze Martin, ${ }^{1}$ Víctor Hugo Olmedo Canchola, ${ }^{2}$ Jéssica Valeria Andoney Mayén ${ }^{3}$
}

\section{INTRODUCCIÓN}

En estos 16 años que han transcurrido desde que inició el siglo XXI, el mundo se ha visto envuelto en una vorágine de avances tecnológicos en todos los rubros de la ciencia y la vida cotidiana.

Las tecnologías de la información y comunicación, conocidas por su abreviatura como TIC, son un conjunto de tecnologías requeridas para el almacenamiento, recuperación, proceso y comunicación de la información. ${ }^{1}$

\section{Antecedentes de las TIC}

La información y comunicación datan de tiempos prehistóricos; un ejemplo de ello son las pinturas rupestres, que revelan la organización de un sistema de transmisión de señales de los habitantes de esas épocas. Estas formas han evolucionado con la creación de nuevas tecnologías, que facilitaron el intercambio de la información. Sin embargo, fue hasta los años 70 cuando inició la "era digital"; los avances científicos en el campo de la electrónica causaron el impulso de las TIC, que combinaban esencialmente la electrónica con el software.

Ya en los años 90, las investigaciones desarrolladas permitieron la convergencia de la electrónica, la informática y las telecomunicaciones, lo que hizo posible la interco-

\footnotetext{
1 Jefe de la Subdivisión de Especializaciones Médicas.

${ }^{2}$ Coordinador de Subcomités Académicos.

${ }^{3}$ Médica pasante en servicio social.
}

División de Estudios de Postgrado, Facultad de Medicina, UNAM.

Correspondencia:

Dr. Víctor Hugo Olmedo Canchola

Correo electrónico: victor.olmedo@fmposgrado.unam.mx vh.olmedo.canchola@gmail.com

Aceptado: 09-03-2017.

Este artículo puede ser consultado en versión completa en http:// www.medigraphic.com/actamedica nexión entre redes, y fue así que surgieron las TIC tal como ahora las conocemos. ${ }^{2}$

\section{Ejemplos y características de las TIC}

Existen múltiples ejemplos de TIC como el teléfono, los celulares, la imprenta, el correo y las computadoras, pero, sin duda, el que ha causado más impacto en el desarrollo de las sociedades es el internet.

El internet es más que una plataforma para el intercambio de datos: es la red mundial que permite el acceso a la información desde cualquier parte del mundo y hace posible la comunicación desde distintos lugares sin la necesidad de estar frente a frente. Todo esto sucede en un lapso casi instantáneo, lo que facilita el intercambio y la obtención de información prácticamente inmediatos.

Algunas de las características fundamentales de las TIC son: ${ }^{3}$

- Inmaterialidad, su materia prima es la información en múltiples códigos y formas.

- Interconexión, aunque se presentan de forma independiente, pueden combinarse y ampliar sus conexiones.

- Interactividad, permite la interacción del sujeto con la máquina y, así, la adaptación de éstas a diversas áreas educativas y cognoscitivas de las personas.

- Instantaneidad, facilita la rapidez de acceso e intercambio de la información.

- Calidad de imagen y/o sonido, lo que da fiabilidad y fidelidad a la información transferida.

- Penetración en diversos sectores de la sociedad: salud, educación, economía, etc.

\section{USO DE LAS TIC EN LA EDUCACIÓN}

Las tecnologías de la información y la comunicación pueden contribuir al acceso universal de la educación, la igualdad, el ejercicio de la enseñanza y el aprendizaje de calidad; facilitan ampliar la información, mejorar la calidad y garantizar la integración (UNESCO, 2015). ${ }^{4}$

La tecnología que influye actualmente en la sociedad representa nuevos retos, especialmente en la educación, 
pues las TIC se han integrado en los procesos educativos de enseñanza-aprendizaje a lo largo del tiempo, generando influencia en la adquisición y el fortalecimiento de los conocimientos por parte de alumnos y profesores.

El impacto de las TIC en la mejoría de la educación depende de diversos factores, pero los que tienen mayor importancia son la accesibilidad a los materiales básicos, tales como una computadora con acceso a internet, y la capacidad del profesor y el alumno para el manejo de estas tecnologías.

La calidad de los docentes y su capacitación profesional permanente es fundamental para lograr la educación de calidad. Con el surgimiento de las TIC, se mediaron los procesos de formación, actualización y capacitación a distancia. Cualquiera que tenga la infraestructura tecnológica puede ingresar a ésta. ${ }^{5}$

\section{LAS TIC EN LA EDUCACIÓN MÉDICA}

\section{Residencias médicas de la UNAM}

La Universidad Nacional Autónoma de México (UNAM), mediante la Facultad de Medicina, otorga el aval universitario a 78 especialidades médicas, todas ellas incluidas en el Plan Único de Especializaciones Médicas (PUEM), con un total de 620 cursos distribuidos en 114 sedes pertenecientes a distintas instituciones de salud.

Las instituciones de salud que cuentan con cursos avalados por la UNAM son el Instituto Mexicano del Seguro Social (IMSS), Instituto de Seguridad y Servicios Sociales de los Trabajadores del Estado (ISSSTE), Petróleos Mexicanos (PEMEX), Secretaría de Salud (SSa) Federal, Secretaría de Salud de la Ciudad de México (SSaCDMX), instituciones privadas, Cruz Roja Mexicana, Instituto de Seguridad Social del Estado de México y Municipios (ISSEMyM), Desarrollo Integral de la Familia (DIF) y UNAM. ${ }^{6}$

La instancia encargada de supervisar, evaluar y apoyar los cursos de postgrado es la Coordinación de Subcomités Académicos, con apoyo de los integrantes de los 46 Subcomités Académicos. Estas supervisiones son con el fin de asegurar que los cursos de especialidad se apegan a los lineamientos que se señalan en el PUEM y cuentan con la infraestructura para la impartición del curso; otros parámetros evaluados son la investigación, los profesores, y la opinión de los alumnos.

En cuanto a infraestructura, durante las visitas se ha documentado que alrededor del $50 \%$ de las sedes no cuentan con acceso a internet inalámbrico libre, o únicamente cuentan con acceso a internet limitado en la biblioteca del hospital. Además, hay escasez de equipos de cómputo para uso de los médicos residentes. Sin embargo, los residentes tratan de subsanar estas deficiencias utilizando sus propios recursos tecnológicos como teléfonos inteligentes, tabletas, computadoras portátiles, e incluso contratando la red inalámbrica externa.

La falta del material básico para poner en práctica las TIC (computadoras con acceso a internet) genera una deficiencia en la aplicación de las nuevas tecnologías para mejorar la enseñanza-aprendizaje de los profesores y residentes. De igual forma, estas deficiencias afectan la aplicación de recursos relacionados con la informática biomédica en las sedes, como son la telemedicina y la educación a distancia (e-learning).

\section{APLICACIÓN DE LAS TIC EN LOS CURSOS DE POSTGRADO DE LA FACULTAD DE MEDICINA}

En la actualidad, uno de los principales retos de la informática clínica es la dificultad que les genera a los profesionales de la salud distinguir entre la informática y la tecnología de la información.

El uso eficaz del conocimiento y la información aplicados a la atención del paciente, la salud pública y la informática clínica no se trata únicamente del empleo de las computadoras en la medicina, sino de un conjunto de conocimientos, métodos y teorías centrados en la utilización eficaz de la información y el conocimiento para mejorar la calidad, la seguridad y el costo-beneficio de la atención del paciente, así como la educación de los médicos residentes y profesores. ${ }^{7}$

Las TIC se pueden utilizar de diversas maneras en los procesos educativos de enseñanza-aprendizaje. Sus diferentes aplicaciones de las TIC servir en los cursos de postgrado de la Facultad de Medicina dependiendo de la habilidad o aprendizaje que se quiere cubrir: ${ }^{8}$

- Medio de expresión: creación de diversas formas para transmitir información. Ejemplos:

- Ponencias: PowerPoint (Microsoft Office), Prezi, SlideShare, PhotoPeach, Flix Time y Padlet. ${ }^{9}$

- Videos: YouTube, EDpuzzle, EduCanon, HapYak, Blubbr y The Mad Video. ${ }^{10}$

- Fuente de información y recursos: obtención de grandes cantidades de información de manera sencilla y rápida. Ejemplos:

- Medios de difusión y almacenamiento: CD/DVD pregrabados, radio, televisión.

- Buscadores: Google Chrome, Internet Explorer, Mozilla Firefox, Opera Mini, iCab, OmniWeb.

- Documentos en la nube: Dropbox, Google Drive, iCloud, WeTransfer, Jumpshare.

- Canal de comunicación: facilitación del intercambio de ideas y documentos, así como del trabajo en equipo entre personas que se encuentran a pequeñas o grandes distancias. Ejemplos: 
- Correo electrónico: Outlook, Hotmail, Yahoo, Gmail, Live e institucionales.

- Mensajería instantánea: WhatsApp, Hangouts, Messenger, Snapchat, Telegram, Line, Skype, Vibre.

- Redes sociales: Facebook, Twitter, Instagram, Blog, Vine, Tumblr.

- Instrumento cognitivo: los elementos de las TIC pueden apoyar el desarrollo de procesos cognitivos, si éste es el fin que se le desea dar.

- Plataformas virtuales: Moodle, Com8s, Schoology, Lectrio, Udemy, Edu 2.0. ${ }^{11}$

- Instrumento de evaluación: evaluación de los conocimientos de las personas de manera rápida, reduciendo el tiempo, incluso a distancia. Se puede seguir el desempeño de cada persona. Ejemplos:

- Evaluaciones en línea: iGiveTest, ExamTime, SurveyMonkey, Respondus, Encuestafácil, E-encuesta. ${ }^{12}$

- Instrumento para procesar información: creación de bases de datos, elaboración de traducciones, realización de cálculos. Ejemplos:

- Crear documentos: Word (Microsoft Office), Power PDF, OfficeSuite Pro 8, Kingston Office. ${ }^{13}$

- Crear hojas de cálculo: OpenOffice Calc, Excel (Microsoft Office), Smartsheet, ThinkFree, Zoho Sheet. ${ }^{14}$

\section{Google en la educación}

Actualmente, uno de los grandes productores de TIC para la educación es la empresa Google, que tiene a disposición de los usuarios diversas aplicaciones que se podrían aplicar en los cursos de especialidades médicas que imparte la Facultad de Medicina. ${ }^{15}$

- Google Classroom: centro de control para las clases; se pueden crear clases, repartir tareas, enviar comentarios y tener acceso a la información de todos los alumnos desde un solo lugar.

Ejemplo: preparar clases en línea para los residentes con el fin de que todos tengan acceso al material y los alumnos que están rotando puedan tener acceso a las clases que se imparten en la sede.

- Google Gmail: correo electrónico.

Ejemplo: se puede crear un correo electrónico en común para residentes y maestros donde se puede enviar información a disposición de todos.

- Google Drive: permite archivar documentos en la nube, acceder a archivos desde cualquier lugar (teléfono inteligente, tableta, computadora), compartir archivos y carpetas, editar documentos.

Ejemplo: se puede utilizar en la elaboración de los protocolos de tesis de los residentes, compartirlos con los tutores en la nube, y ambos pueden realizar cambios a los textos, guardar la información consultada, compartir artículos, casos clínicos, etc.

- Google Calendar: calendario en línea donde se pueden agregar reuniones, citas, recordatorios, y tenerlo disponible en cualquier momento.

Ejemplo: se pueden programar las clases de los residentes, vacaciones, rotaciones, poner recordatorios de sesiones académicas o bibliográficas y compartirlo con todo el equipo de trabajo.

- Google Docs: da la posibilidad de crear, compartir y acceder en línea a los documentos desde cualquier lugar; administrar documentos, hojas de cálculo, presentaciones, encuestas, etcétera.

Ejemplo: elaborar presentaciones o documentos desde cualquier dispositivo con acceso a internet sin la necesidad de tener instalada alguna paquetería para edición de documentos.

- Google Sheets: colabora, crea o edita junto con otros usuarios hojas de cálculo desde cualquier lugar.

Ejemplo: elaborar las rotaciones, distribuir temas, programar las vacaciones de los residentes en colaboración con toda la plantilla de profesores.

- Google Slides: colabora, crea o edita junto con otros usuarios presentaciones desde cualquier lugar.

Ejemplo: elaborar presentaciones por grupos de residentes para exponer algún caso clínico o revisión de tema. Fomenta el trabajo en equipo.

- Google Sites: crear sitios web para un equipo de trabajo donde pueden recopilar información de diversos tipos y compartir con algún grupo.

Ejemplo: elaborar una plataforma virtual para profesores y residentes donde esté disponible información sobre algún tema o caso clínico; se pueden realizar tareas y evaluaciones para el seguimiento de los residentes, así como compartir enlaces sobre congresos, videos, o artículos.

- Google Hangouts: mensajería instantánea, llamadas de voz, videollamadas.

- Ejemplo: el profesor titular puede establecer un grupo de chat o un videochat para enviar lecciones en línea a los residentes, crear clases o un grupo para intercambio de opiniones (discusión de casos).

\section{Ventajas y desventajas de las TIC en los cursos de} postgrado de la Facultad de Medicina ${ }^{16}$

Las TIC fueron creadas con el fin de ser herramientas valiosas para la materialización del conocimiento que adquieren los residentes.

"Los rápidos progresos de las tecnologías de la información y la comunicación modifican la forma de elaboración, adquisición y transmisión de conocimientos" (UNESCO, 1998). 


\section{Ventajas}

- Propician y mantienen el interés, motivación e interacción de los residentes y profesores.

- Desarrollan la iniciativa y el aprendizaje a partir de errores.

- Fomentan la comunicación entre profesores y residentes.

- Fortalecen las habilidades de búsqueda y selección de información.

- Contribuyen con una mayor fuente de recursos informativos.

- Facilitan la evaluación y el seguimiento de los residentes.

- Atienden diferentes estilos de aprendizaje.

- Promueven el aprendizaje autorregulado por parte de los residentes.

- Impulsan el trabajo y aprendizaje cooperativo entre residentes y profesores.

\section{Desventajas}

- Pueden producir estrés por desconocimiento.

- Desarrollan dependencia a los sistemas informáticos.

- Se necesitan equipos de computación con acceso a internet libre.

- Puede haber problemas propios del equipo de cómputo.

\section{CONCLUSIONES}

Las tecnologías de la información y comunicaciones han renovado la educación internacionalmente; son herramientas valiosas de información y medios para facilitar el proceso enseñanza-aprendizaje tanto de alumnos como de profesores.

El uso de las TIC en la educación médica es una herramienta esencial para mejorar la calidad de educación y el adiestramiento de los residentes en formación de las distintas sedes que avala la Facultad de Medicina por parte de la UNAM. Sin embargo, una de las principales limitantes a las que se enfrentan los residentes es la falta de equipos de cómputo con acceso a internet para su uso académico, lo que facilitaría el acceso a artículos recientes, guías de práctica clínica que apoyen el abordaje y tratamiento de los pacientes, plataformas académicas, entre otros.

Por lo anterior, se insiste en la necesidad de proporcionar los recursos didácticos y tecnológicos necesarios para lograr un mejor aprendizaje y aprovechamiento de los residentes en los cursos de especializaciones médicas.

\section{REFERENCIAS}

1. Belloch C. Las tecnologías de la información y comunicación en el aprendizaje. Material docente [en línea]. Universidad de Valencia, Departamento de Métodos de Investigación y Diagnóstico en Educación; 2012. Disponible en: http://www.uv.es/bellochc/ pedagogia/EVA1.pdf

2. Tecnologías de la información y las comunicaciones. [Consultado el 25 de octubre de 2016]. Disponible en: https://sites.google.com/site/ ticsyopal5/assignments

3. Cabero J. Las aportaciones de las nuevas tecnologías a las instituciones de formación continuas: reflexiones para comenzar el debate. En: Departamento de Didáctica y Organización Escolar, Universidad Complutense-UNED: Las organizaciones ante los retos del siglo XXI. 1998. pp. 1143-1149. [Consultado el 26 de octubre de 2016]. Disponible en: http://tecnologiaedu.us.es/cuestionario/bibliovir/85.pdf

4. Las tecnologías de la información y comunicaciones (TIC) en la educación. [Consultado el 26 de octubre de 2016]. Disponible en: http://www.unesco.org/new/es/unesco/themes/icts/

5. Florina GL, Argelia RV. E-learning en la educación médica. Rev Fac Med UNAM [Internet]. 2012 [Consultado el 26 de octubre de 2016]; 55 (2): 27-37. Disponible en: http://www.medigraphic.com/pdfs/ facmed/un-2012/un122e.pdf

6. Documentos técnicos de la División de Estudios de Posgrado, UNAM.

7. Detmer DE, Shortliffe EH. Clinical informatics: prospects for a new medical subspecialty. JAMA. 2014; 311 (20): 2067-2068.

8. Las TIC en la educación. [Consultado el 26 de octubre de 2016]. Disponible en: http://www.cursosinea.conevyt.org.mx/para_asesor/ tics/tema1_2.htm

9. Cinco herramientas para hacer presentaciones 2.0. [Consultado el 26 de octubre de 2016]. Disponible en: http://www.aulaplaneta. com/2014/06/17/recursos-tic/cinco-herramientas-para-hacerpresentaciones-2-0/

10. Cinco herramientas para crear videos interactivos. [Consultado el 26 de octubre de 2016]. Disponible en: http://www.aulaplaneta. com/2015/01/28/recursos-tic/cinco-herramientas-para-crear-videosinteractivos/

11. Plataformas virtuales educativas gratuitas. [Consultado el 26 de octubre de 2016], Disponible en: http://www.ticeducacionec. com/2014/05/30-plataformas-virtuales-educativas.html

12. Treinta herramientas para crear evaluaciones, test $y /$ o encuestas onlinedescargables. [Consultado el 27 de octubre de 2016]. Disponible en: http://www.ticeducacionec.com/2014/03/herramientas-para-crearevaluaciones.html

13. Quince aplicaciones para crear y modificar documentos. [Consultado el 27 de octubre de 2016]. Disponible en: http://www. educaciontrespuntocero.com/recursos/aplicaciones-para-crearmodificar-documentos/18847.html

14. Cinco programas de hojas de cálculo. [Consultado el 27 de octubre de 2016]. Disponible en: http://hojasdecalculo.about.com/od/Excel/ tp/5-Programas-de-hojas-de-caacutelculo-gratis.htm

15. Google for Education. Education On Air. [Consultado el 28 de octubre del 2016]. Disponible en: https://www.google.com/intl/es_es/edu/ products/productivity-tools/

16. Castro S, Guzmán B, Casado D. Las TIC en los procesos de enseñanza y aprendizaje. Laurus [Internet]. 2007 [Consultado el 28 de octubre de 2016]; 13 (23): 213-234. Disponible en: http://google.redalyc. org/articulo.oa?id $=76102311$ 